\title{
Detection of Liver Fibrosis with Magnetic Cross-Relaxation
}

\author{
Alex M. Aisen, Kei Doi, Scott D. Swanson
}

The utility of MRI using magnetization transfer (MT) enhanced pulse sequences to diagnose hepatic cirrhosis in a rat model was investigated. Hepatic $T_{1}$ was measured with and without MT off-resonance RF pulses in 17 treated and six control rats. The livers were evaluated histologically, and the hydroxyproline content quantitatively measured. We did not find a statistically significant linear correlation between the MR relaxation times and the degree of tissue injury. However, the MR measurements performed with MT were superior to those without differentiating the treated and control groups. Specifically, the $T_{1}$ times were $695 \pm 76 \mathrm{~ms}$ for the treated group, versus $748 \pm 61 \mathrm{~ms}$ in the controls; $P=0.095$. The $T_{1 \text { sat }}$ times were also lower in the treated group, with statistical significance: $367 \pm 51 \mathrm{~ms}$ versus $421 \pm 38 \mathrm{~ms}, P=0.016$. Finally, the change in the relaxation rates (the inverse of the relaxation times) with and without saturation were $1.31 \pm 0.22$ $\mathrm{s}^{-1}$ (treated group) versus $1.05 \pm 0.12 \mathrm{~s}^{-1}$ (controls), which differed significantly, $P=0.001$.

Key words: cirrhosis; fibrosis; collagen; MTC.

\section{INTRODUCTION}

The specific identification of fibrosis is an important problem in imaging diagnosis. X-ray and ultrasound based imaging have not been able to make this differentiation; conventional MRI has also been disappointing (1-6).

The liver provides a useful organ for study. Clinically, many diseases are characterized by inflammation and edema which may progress to fibrotic scarring, or cirrhosis. Diagnosis of cirrhotic change by noninvasive means would be valuable. Long-term administration of the solvent carbon tetrachloride to rats is known to produce a condition which in many ways mimics many human diseases (7). In earlier work using this model, Chamuleau et al. found that hepatic $T_{1}$ levels rose with liver injury, peaking at 4 weeks and maintaining this plateau; collagen levels reached their maximum at 8 weeks. They concluded that the $T_{1}$ increases represented edema, and were not useful for measuring fibrosis (2).

Magnetization transfer contrast (MTC) is a technique where RF radiation is employed to selectively saturate the nuclear magnetism of immobile protons in membranes or macromolecules such as collagen (8-15). We

MRM 31:551-556 (1994)

From the Department of Radiology, The University of Michigan, Ann Arbor, Michigan.

Address correspondence to: Scott D. Swanson, Ph.D., Department of Radiology, University of Michigan Hospitals, R3301 Kresge III/Box 0553, Ann Arbor, Ml 48109-0553.

Received September 10, 1993; revised January 20, 1994; accepted January $20,1994$.

0740-3194/94 $\$ 3.00$

Copyright (c) 1994 by Williams \& Wilkins

All rights of reproduction in any form reserved.

investigated the use of MTC to determine if the technique might allow differentiation of tissue fibrosis from other pathology, and to assess its potential to quantify the amount of fibrous tissue present.

The basic hypothesis of this work is that as the amount of solid material, specifically collagen, increases in the liver with chemically induced fibrosis, the cross relaxation pathway will become a more dominant mechanism for $T_{1}$ in the tissue and shorten the measured $T_{1}$ in tissue. Observation of this phenomenon in the liver is complicated by edema, which also results from the carbon tetrachloride treatment. Hepatic edema will act conversely to lengthen the measured $T_{1}$. These competing effects complicate efforts to measure liver fibrosis with conventional MRI.

Off-resonance $R F$ irradiation selectively saturates the immobile proton magnetization of the tissue and indirectly, via magnetic cross relaxation, saturates the MRI detected, mobile proton magnetization $(9,10)$. In addition, indirect $R F$ saturation also decreases the apparent $T_{1}$ of the mobile proton magnetization to a value defined as $T_{1 \text { sat }}(8)$. It was our hope that measurement of these parameters before and after MTC irradiation would provide specific information on the presence of cirrhosis.

\section{METHODS}

\section{Treatment Protocol}

White Sprague-Dawley rats received a 3-week course of phenobarbital (to induce hepatic enzyme systems), followed by 14 weeks of administration of carbon tetrachloride by NG tube. Following initial dosing with standardized quantities, subsequent doses of $\mathrm{CCl}_{4}$ for each rat were individually determined based on the weight loss which followed previous doses, an approach described by Proctor and Chatamra (7). Twenty-five rats entered the treatment protocol, but only 17 completed it; these form the basis of this report. (Of the remaining eight animals, four died, three did not undergo post-mortem MRI evaluation; and in one rat there was a technical failure in the non-MTC MRI measurements). Six untreated rats served as controls for the MR experiments; however, these control rats did not have histologic and biochemical evaluation. Histologic and biochemical measurements were made on a separate group of 10 untreated rats.

\section{MRI Measurements}

MRI was performed with a $2.0 \mathrm{~T}$ Omega CSI system (Omega software version 6.02) manufactured by Bruker Instruments (Fremont, CA) and equipped with S-180 actively shield gradients. The RF probe for transmit and receive was a home-built saddle coil with an 8-cm diameter and $13-\mathrm{cm}$ length. This probe provided sufficient RF 
homogeneity and sensitivity for the rat liver studies presented here.

The control and treated animals were anaesthetized with ketamine and Rompain. The liver was located with a multislice $T_{1}$-weighted imaging sequence.

Measurements of $T_{1}$ and $T_{1 \text { sat }}$ were made using a single axial slice, 8-point, Look and Locker imaging pulse sequence $(16,17)$. Data was acquired both with and without MTC irradiation, consisting of a $530 \mathrm{~Hz}\left(\gamma B_{1} / 2 \pi\right)$ pulse applied $10 \mathrm{kHz}$ off resonance during the relaxation delay periods of the sequence. The non-MTC and MTC measurements (the latter herein identified with the suffix "sat") were made both in the living animals, and following sacrifice with anesthetic overdose. Respiratory gating or compensation was not used. The postsacrifice measurements are presented here.

The slice-selective Look and Locker sequence is shown in Fig. 1. The magnetization is inverted by a composite $180^{\circ}$ pulse and then sampled a series of by a small flip angle pulses. The measured relaxation rate will be greater than the true relaxation rate due to the sampling of the longitudinal magnetization by the pulse train. Therefore, to obtain $R_{1}$ or $R_{1 \text { sal }}$, a factor of $\ln \operatorname{Cos}(\alpha) / \tau$, where $\tau$ is the

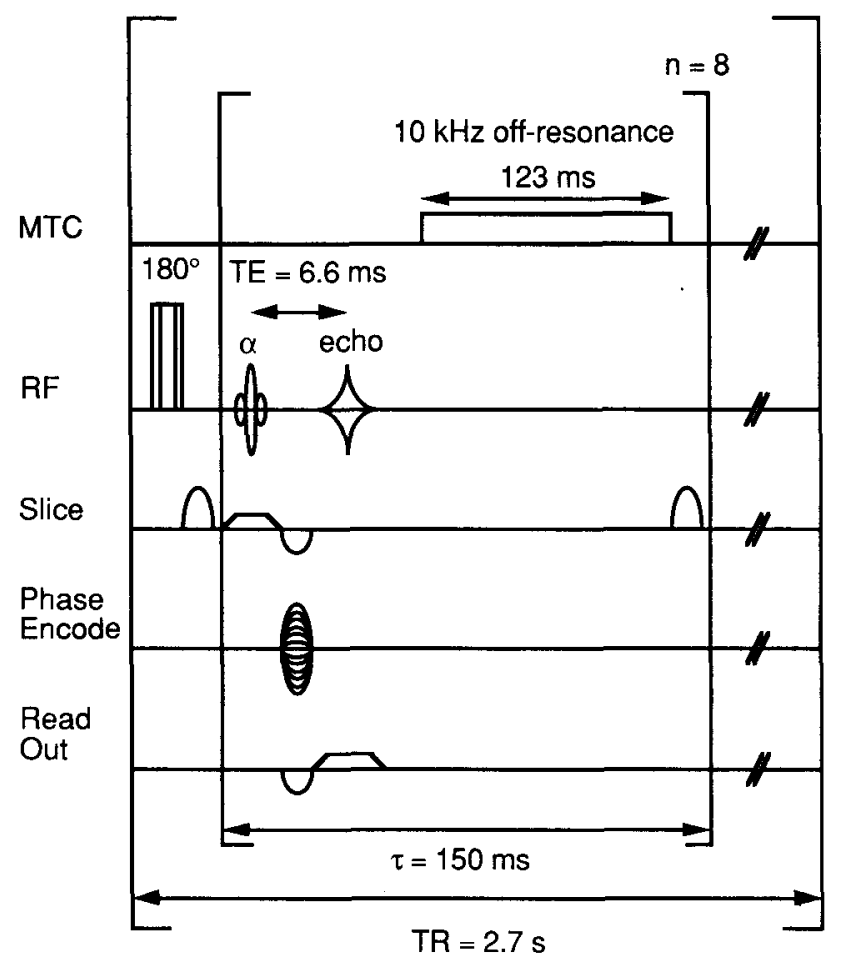

FIG. 1. Look and Locker imaging sequence for determination of $T_{1}$ and $T_{1 \text { sat }}$. Data from eight points along the relaxation curve with a spacing of $150 \mathrm{~ms}$ were collected for each phase encoding line with a TR of $2.7 \mathrm{~s}$, a TE of $6.6 \mathrm{~ms}$, a nominal flip angle $\alpha$ of $15^{\circ}$, a block size of $128 \times 128$, a slice thickness of $5 \mathrm{~mm}$, a FOV of 60 $\mathrm{mm}$, and 2 averages. The MTC pulse was applied $10 \mathrm{kHz}$ offresonance with an amplitude $\gamma B_{1} / 2 \pi$ of $530 \mathrm{~Hz}$ and a duration of $123 \mathrm{~ms}$ for each of the eight relaxation delays. The duty cycle of off-resonance RF irradiation for the entire pulse sequence was $36 \%$. The duty cycle of RF irradiation during the collection of one phase encoding line was $81 \%$, with the remaining time used for frequency shifting and data collection. separation between the pulses and $\alpha$ is the flip angle, must be added to the measured relaxation rates to obtain the true relaxation rates. In the experiments performed here, $\alpha$ was nominally $15^{\circ}$ and $\tau 150 \mathrm{~ms}$, yielding a correction factor of $-0.23 \mathrm{~s}^{-1}$. $T_{1 \mathrm{sat}}$ is determined with the off-resonance RF irradiation turned on during the recovery period. The following MRI parameters were calculated: $T_{1}, T_{1 \text { sat }}$, their inverses (the relaxation rates $R_{1}$ and $R_{1 \text { sat }}$ ), the difference of the relaxation rates, $M_{0}, M_{\mathrm{sat}}$, and the ratio $M_{\text {sat }} / M_{0}$.

Calibration of RF power is critical for the success of this experimental protocol. Knowledge of $\alpha$ is required for the Look and Locker experiment and $T_{1 \text { sat }}$ is a function of the power of the off-resonance RF irradiation. To calibrate the RF power for each experiment, a one-dimensional, spin-echo imaging sequence was used. Specifically, the phase encode gradient was turned off and the power of the $\theta$ and $2 \theta$ pulses in the spin echo sequence varied to maximize the amplitude of the detected echo. The amount of power required to achieve flip angles of $90^{\circ}$ and $180^{\circ}$ was determined and used to normalize the amplitude of the $\alpha$ and MTC pulses in the Look and Locker sequence over the course of this study.

Since the sign of the magnetization changes from negative to positive during the $T_{1}$ inversion-recovery experiment, it is desirable to process the data in a phase sensitive manner. To accomplish this, the following protocol was used. Each of the eight $128 \times 128$ raw $k$-space datasets from an inversion-recovery imaging experiment was baseline corrected and Fourier transformed along both dimensions without taking the magnitude. The 8-phase sensitive images were then divided by the image from the initial time point and multiplied by $\mathbf{- 1}$. This process eliminates phase due to all sources except the inversionrecovery of magnetization and allows us to calculate $T_{1}$ and $T_{1 \text { sat }}$ in a phase sensitive manner. A region-of-interest within the liver was chosen for analysis based on location and signal homogeneity, minimizing arefacts due to RF inhomogeneity and partial voluming. A representative image is shown in Fig. 2. The signal within the ROI, nominally 400 pixels, was summed for each of the eight images and the resulting values used for the determination of $T_{1}$ and $T_{1 \text { sar. }}$ Inversion-recovery data and fitted results for $T_{1}$ and $T_{1 \text { sat }}$ are shown in Fig. 3 .

At the conclusion of the experiments, the livers were removed from all treated and control animals. The organs were divided. Following a period of frozen storage, objective, biochemical assays of hydroxyproline content were performed on one portion of the liver using a method previously described (18). The other portions of the organs were histologically graded on a scale of 0 (normal) through 5 (severe fibrotic change) by an independent animal pathologist blinded to the NMR data. Photomicrographs of liver sections from normal and treated animals are shown in Figs. 4a and 4b, respectively. Wet weight/dry weight was measured following vacuum desiccation of one of the portions of each liver.

Data is expressed as mean \pm standard deviations. Statistical comparisons employed an unpaired, two-tailed Student's $t$ test with unequal variances; the standard for significance is $P<0.05$. 


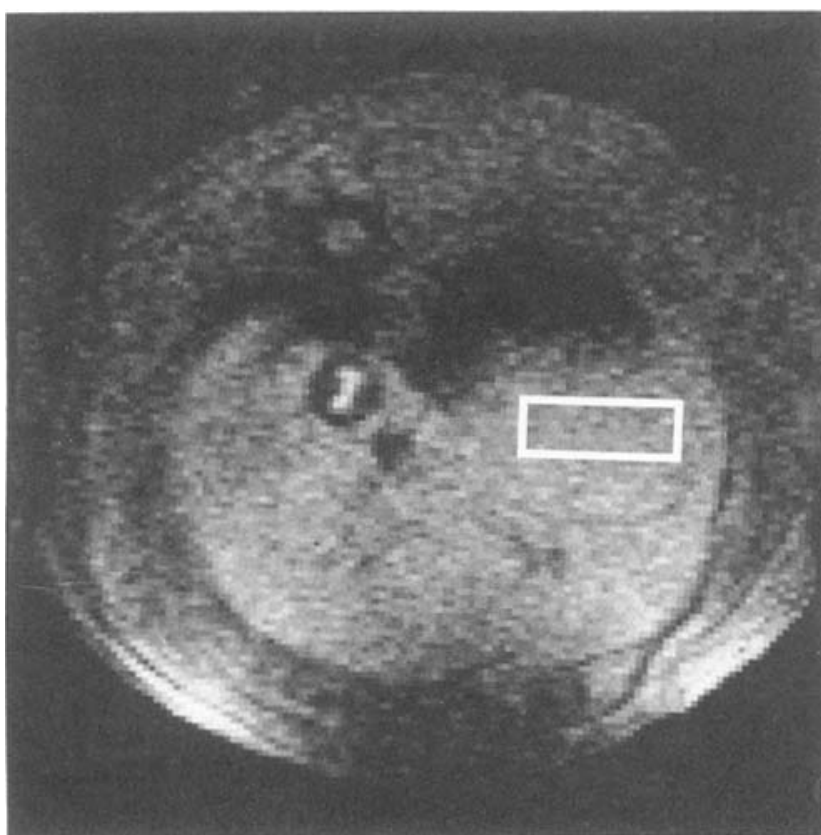

FIG. 2. The eighth image (final time point) acquired with the Look and Locker pulse sequence shown in Fig. 1 with parameters as given in the legend to Fig. 1. Also shown is the ROl from which the data is obtained to produce the relaxation curves in Fig. 3 .

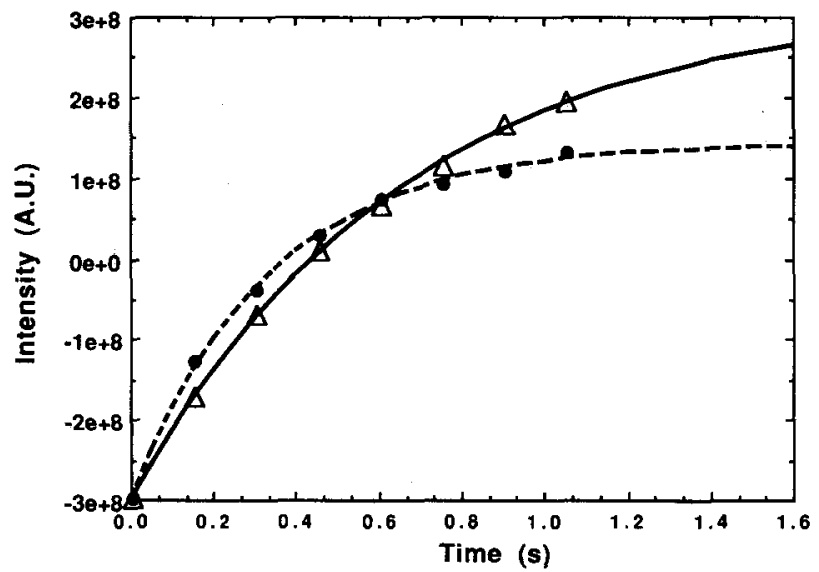

FIG. 3. Data for determination of $T_{1}$ and $T_{1 \text { sat }}$ and fitted relaxation curves. The data for $T_{1}(\Delta)$ and $T_{1 \text { sat }}(\Theta)$ were obtained by summing the signals within the ROI shown in Fig. 2. For this example, the estimates for $T_{1}$ and $T_{1 \text { sar }}$, corrected for flip angle and delay time, are $731 \pm 33 \mathrm{~ms}$ and $350 \pm 17 \mathrm{~ms}$, respectively. The relaxation curves for $T_{1}$ and $T_{1 \text { sat, }}$, calculated with the parameters obtained by the fitting procedure, are indicated by the solid and dashed lines, respectively.

\section{RESULTS}

The presacrifice MR measurements showed considerable scatter when compared with the postmortem measurements. For this reason, final data analysis was performed on the postmortem MRI measurements only, as it was felt that the presacrifice measurements were degraded by inaccuracies resulting from respiratory motion. As noted above, normal values for hydroxyproline content, and histology were obtained in 10 rats; these are different

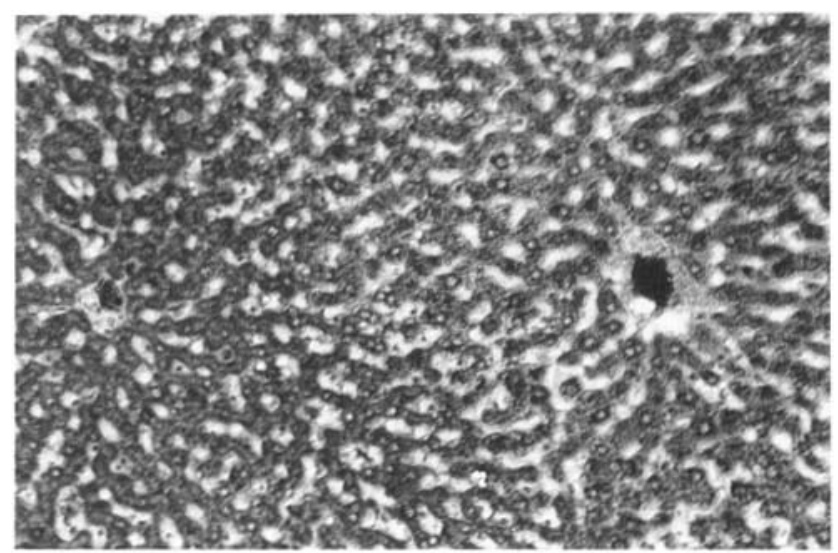

a

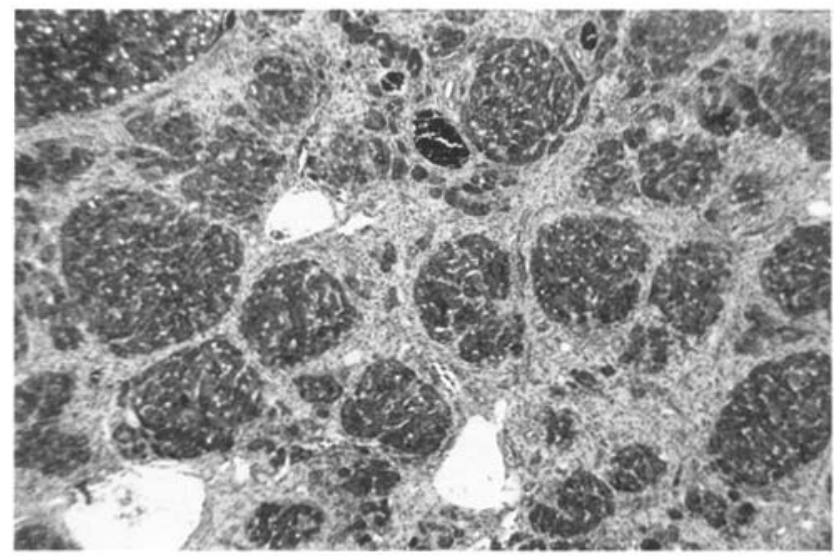

b

FIG. 4. Representative histological specimens (100x) of liver sections from normal (a) and treated (b) animals; the treated animal shown had severe disease, grade 5 .

animals from the six used for the MRI measurements and percent water determinations.

The percent water by weight in the treated animals was $79.7 \% \pm 0.19 \%$, versus $70.9 \% \pm 2.5 \%$ in the six controls. The percent dry weight hydroxyproline content was higher in the treated group, $1.99 \pm 0.81 \mathrm{mg} / \mathrm{gm}$ versus $0.854 \pm 0.12 \mathrm{mg} / \mathrm{g}(P=0.000003518)$. All untreated animals were graded as normal histology; the average grade for the treated animals was 2.8 ( 5 being most severe).

The corrected $T_{1}$ times for the treated animals were lower in the treated animals than the controls, though the difference was not significant: $695 \pm 76 \mathrm{~ms}$ versus $748 \pm$ $61 \mathrm{~ms} ; P=0.095$. The $T_{1 \text { sat }}$ times were also lower in the treated group, with statistical significance: $367 \pm 51 \mathrm{~ms}$ versus $421 \pm 38 \mathrm{~ms}, P=\mathbf{0 . 0 1 6}$.

The change (i.e., difference) of the relaxation rates with and without saturation, $1.31 \pm 0.22 \mathrm{~s}^{-1}$ (treated group) versus $1.05 \pm 0.12 \mathrm{~s}^{-1}$ (controls), differed significantly, $P=0.001$. The ratio of $M_{\text {sat }} / M_{\mathrm{o}}$ differed between the two groups as well; $0.519 \pm 0.029$ for the treated animals versus $0.542 \pm 0.018$ for the controls; $P=\mathbf{0 . 0 2 8}$.

Linear correlations between the MRI parameters and the quantitative hydroxyproline content or histological grades were not revealing.

Similar trends in the $T_{1}$ and $T_{1 \text { sat }}$ differences between 
the groups were observed in the presacrifice data, but these did not reach statistical significance, presumably because of motion.

\section{DISCUSSION}

The histologic determinations revealed substantial variation in the degree of fibrotic change. No statistically significant difference was found between the postsacrifice $T_{1}$ of a group of 17 treated animals and the $T_{1}$ of 6 control animals. However, the $T_{1 \text { sat }}$ measurements did show a significant difference between the treated group and the control group. Interestingly, a strong statistical difference, $P=0.001$, was found in the change in relaxation rates which occurred with application of MTC saturation (i.e., $R_{1 \text { sat }}-R_{1}$ ) when the treatment and control groups were compared. These results are encouraging, but by no means conclusive. By providing an additional mechanism for relaxation to occur, MTC irradiation is expected to shorten $T_{1}$ times; this effect will be most pronounced when there is a substantial amount of fibrosis tissue present. Our results are consistent with this. We had hoped to be able to use this effect to quantitate the amount of fibrous tissue present, but were unsuccessful: correlations of the relaxation parameters with the quantitative measures of fibrosis in a pooled group including both treated and untreated animals was not revealing. We cannot tell whether this is a result of inaccuracies in our measurements or an intrinsic limitation of the MTC technique.

The data presented here describes post-mortem measurements obtained on both treated and control animals; data collected in living animals showed similar trends, but did not reach statistical significance. Perfusion has been shown to slightly alter MTC measurements (19). Thus, it is conceivable that alterations in blood perfusion in living animals with cirrhosis might affect measurements such as ours. This possibility could be investigated in future work.

In contrast to Chamuleau et al., we found that the $T_{1}$ relaxation times in our treated animals decreased. The difference is almost certainly a result of the longer treatment period we used compared with Chamuleau; this would be expected to allow hepatic inflammation to progress from edema towards end-stage fibrotic cirrhosis. This is largely substantiated by our histologic and percent water data. Chamuleau et al. made their measurements on living animals using respiratory gating; our data are postmortem.

The identification of fibrosis is an important clinical problem. In some cases, such as treated malignancies, it is necessary to differentiate recurrent or residual neoplasm from fibrous scarring. In other conditions, such as parenchymal diseases of the liver and kidney, the presence of fibrous tissue is usually an indicator of end-stage disease which is unlikely to respond to aggressive therapy, while the absence of fibrosis has opposite implications. Pharmacological therapies are becoming available which may be able to retard or prevent fibrosis (20-24); the early detection of fibrotic change, and the ability to monitor it over time seem crucial to the use of such therapy. In pulmonary disease, it is often difficult to differentiate interstitial water or edema from fibrosis, though the two conditions have completely different implications and therapies.

Presently, biopsy is considered the only reliable means of diagnosing fibrotic change, though serial biochemical assays of serum procollagen levels have been proposed for liver disease (25). MR has been investigated as a potential tool for quantitatively assessing fibrosis, although the results have thus far been discouraging. As discussed earlier, Chamuleau et al. concluded that changes in $T_{1}$ times reflected edema, and were not useful for detecting fibrosis (2). Similarly, Goldberg et al. found no change in $T_{1}$ and $T_{2}$ times with thioacetamide induced cirrhosis in rats (6), and Rademaker et al. found no change in $T_{1}$ values in human patients with methotrexate-induced cirrhosis (26).

Data for the lung has been more promising, though by no means conclusive. Vinitski et al. documented decreased $T_{1}$ and $T_{2}$ values in lung samples obtained from rats following long-term bleomycin treatment (27). Shioya et al. documented a decrease in the $T_{1}$ (but not $T_{2}$ ) time of lung tissue with late stage radiation induced fibrosis in rats (1). Taylor et al. found an increase in $T_{2}$, but no change in $T_{1}$, in the lung in mice treated with bleomycin (3). Contrast agents may also be helpful (28).

Imaging data has been reported in humans, with attempts to correlate appearance on $T_{2}$-weighted images with fibrotic scarring, since fibrous tissue is felt to have a short $T_{2}$ time. This has yielded very mixed results; most investigators find fibrous tissue to be dark on $T_{2}$ weighted images (29-33), although the results are by no means always consistent; Negandank et al., for example, found that dense fibrosis in patients with histories of lymphoma was bright on $T_{2}$-weighted images (5).

Many (34-37), but not all $(26,38)$, studies suggest that MRI and contrast-enhanced MRI $(39,40)$ may be of use in diagnosing diffuse hepatic disease, including hepatitis with edema; however, there is little evidence that conventional MRI is useful in diagnosing fibrotic change. Similarly, MR spectroscopy (41-43) techniques offer promise for characterization of liver disease, including impairment in hepatocellular function, but has not yet been shown to be specific for fibrosis.

The data presented here is limited by the accuracy of the MR measurements, even though postmortem data free from motion artifact is used in the analysis. It seems likely that in vivo measurements will require an alternative approach, which is more motion insensitive that our technique. One possibility is a spin-echo technique employing column selection based on the intersection of orthogonal slice selective $90^{\circ}$ and $180^{\circ}$ pulses, with a read-out gradient applied along the column axis.

\section{CONCLUSION}

The results are encouraging but not conclusive. The increase in $R_{1 \text { sat }}$ for fibrotic livers was reliably seen only in the data from the sacrificed animals and therefore motion insensitive pulse sequences would probably be helpful to measure $R_{1 \text { sat }}$ in vivo. An increase in the $R_{1}$ relaxation rate 
for fibrotic livers compared with control livers is consistent with an increase in the fraction of macromolecular protons (e.g., collagen). The relaxation rates measured here are, however, a complicated function of the many tissue constituents and the change seen is not necessarily due to the increased collagen levels. Nonetheless, this study suggests that $R_{1 \text { sat }}$ is sensitive to the solid content in tissue and may be helpful in the diagnosis of liver fibrosis.

\section{ACKNOWLEDGMENTS}

The authors thank Dr. Sem Phan for performing the hydroxyproline assays, and the wet/dry weight determinations, and Nancy Love, DVM, for her help with preliminary experiments.

\section{REFERENCES}

1. S. Shioya, M. Haida, M. Fukuzaki, Y. Ono, M. Tsuda, Y. Ohta, H. Yamabayashi, A 1-year time course study of the relaxation times and histology for irradiated rat lungs. Magn. Reson. Med. 14, 358-368 (1990).

2. R. A. Chamuleau, J. H. Creyghton, I. De Nie, M. A. Moerland, O. R. Van der Lende, J. Smidt, Is the magnetic resonance imaging proton spin-lattice relaxation time a reliable noninvasive parameter of developing liver fibrosis? Hepatology 8, 217-221 (1988).

3. C. R. Taylor, H. D. Sostman, J. C. Gore, G. W. Smith, Proton relaxation times in bleomycin-induced lung injury. Invest. Radiol. 22, 621-626 (1987).

4. J. K. Lee, H. S. Glazer, Controversy in the MR imaging appearance of fibrosis [editorial; comment]. Radiology 177, 21-22 (1990).

5. W. Negendank, A. al-Katib, C. Karanes, M. Smith, Lymphomas: MR imaging contrast characteristics with clinicalpathologic correlations. Radiology 177, 209-216 (1990).

6. H. I. Goldberg, A. A. Moss, D. D. Stark, J. McKerrow, B. Engelstad, A. Brito, Hepatic cirrhosis: magnetic resonance imaging. Radiology 153, 737-739 (1984).

7. E. Proctor, K. Chatamra, Controlled induction of cirrhosis in the rat. Br. J. Exp. Pathol. 64, 320-330 (1983).

8. H. N. Yeung, S. D. Swanson, Transient decay of longitudinal magnetization in heterogeneous spin systems under selective saturation. I. Magn. Reson. 99, 466-479 (1992).

9. J. Grad, D. Mendelson, F. Hyder, R. G. Bryant, Applications of nuclear magnetic cross-relaxation spectroscopy to tissues. Magn. Reson. Med. 17, 452-459 (1991).

10. S. D. Wolff, R. S. Balaban, Magnetization transfer contrast (MTC) and tissue water proton relaxation in vivo. Magn. Reson. Med. 10, 135-144 (1989).

11. R. S. Balaban, S. Chesnick, K. Hedges, F. Samaha, F. W. Heineman, Magnetization transfer contrast in MR imaging of the heart. Radiology 180, 671-675 (1991).

12. R. S. Balaban, T. L. Ceckler, Magnetization transfer contrast in magnetic resonance imaging. Magn. Reson. Q. 8, 116-137 (1992).

13. S. D. Wolff, R. S. Balaban, Magnetization transfer contrast (MTC) and tissue water proton relaxation in vivo. Magn. Reson. Med. 10, 135-144 (1989).

14. S. D. Wolff, S. Chesnick, J. A. Frank, K. O. Lim, R. S. Balaban, Magnetization transfer contrast: MR imaging of the knee. Radiology 179, 623-628 (1991).

15. S. D. Wolff, J. Eng, R. S. Balaban, Magnetization transfer contrast: method for improving contrast in gradient-recalled-echo images [see comments]. Radiology 179, 133-137 (1991).
16. D. C. Look, D. R. Locker, Time saving in measurement of NMR and EPR relaxation times. Rev. Sci. Instrum. 41, 250251 (1970).

17. Y. T. Zhang, H. N. Yeung, P. L. Carson, J. H. Ellis, Experimental analysis of T1 imaging with a single-scan, multiplepoint, inversion-recovery technique. Magn. Reson. Med. 25, 337-343 (1992).

18. S. H. Phan, D. Schrier, B. McGarry, R. E. Duque, Effect of the beige mutation on bleomycin-induced pulmonary fibrosis in mice. Am. Rev. Respir. Dis. 127, 456-459 (1983).

19. P. V. Prasad, D. Burstein, R. R. Edelman, MRI evaluation of myocardial perfusion without a contrast agent using magnetization transfer. Magn. Reson. Med. 30, 267-270 (1993).

20. D. Kershenobich, F. Vargas, G. Garcia-Tsao, R. Perez Tamayo, M. Gent, M. Rojkind, Colchicine in the treatment of cirrhosis of the liver. N. Engl. J. Med. 318, 1709-1713 (1988).

21. C. McClurkin, Jr., S. H. Phan, C. H. Hsu, S. R. Patel, J. K. Spicker, A. M. Kshirsagar, W. Y. Yuan, R. C. Wiggins, Moderate protection of renal function and reduction of fibrosis by colchicine in a model of anti-GBM disease in the rabbit. J. Am. Soc. Nephrol. 1, 257-265 (1990).

22. E. R. Schiff, Hepatic fibrosis-new therapeutic approaches [editorial; comment]. N. Engl. J. Med. 324, 987-989 (1991).

23. R. H. Wiesner, J. Ludwig, K. D. Lindor, R. A. Jorgensen, W. P. Baldus, H. A. Homburger, E. R. Dickson, A controlled trial of cyclosporine in the treatment of primary biliary cirrhosis [see comments]. N. Engl. J. Med. 322, 1419-1424 (1990).

24. M. M. Kaplan, D. W. Alling, H. J. Zimmerman, H. J. Wolfe, R. A. Sepersky, G. S. Hirsch, G. H. Elta, K. A. Glick, K. A. Eagen, A prospective trial of colchicine for primary biliary cirrhosis. N. Engl. J. Med. 315, 1448-1454 (1986).

25. M. J. Ruwart, K. F. Wilkinson, B. D. Rush, T. J. Vidmar, K. M. Peters, K. S. Henley, H. D. Appelman, K. Y. Kim, D. Schuppan, E. G. Hahn, The integrated value of serum procollagen III peptide over time predicts hepatic hydroxyproline content and stainable collagen in a model of dietary cirrhosis in the rat [see comments]. Hepatology 10, 801-806 (1989).

26. M. Rademaker, J. A. Webb, D. G. Lowe, R. H. MeyrickThomas, J. D. Kirby, D. D. Munro, Magnetic resonance imaging as a screening procedure for methotrexate induced liver damage. Br. J. Dermatol. 117, 311-316 (1987).

27. S. Vinitski, M. G. Pearson, S. J. Karlik, W. K. Morgan, L. S. Carey, G. Perkins, T. Goto, D. Befus, Differentiation of parenchymal lung disorders with in vitro proton nuclear magnetic resonance. Magn. Reson. Med. 3, 120-125 (1986).

28. Y. Berthezene, V. Vexler, R. Kuwatsuru, W. Rosenau, A. Muhler, O. Clement, D. C. Price, R. C. Brasch, Differentiation of alveolitis and pulmonary fibrosis with a macromolecular MR imaging contrast agent. Radiology 185, 97-103 (1992).

29. G. P. Krestin, W. Steinbrich, G. Friedmann, Recurrent rectal cancer: diagnosis with MR imaging versus CT. Radiology 168, 307-311 (1988).

30. H. S. Glazer, R. G. Levitt, J. K. Lee, B. Emani, S. Gronemeyer, W. A. Murphy, Differentiation of radiation fibrosis from recurrent pulmonary neoplasm by magnetic resonance imaging. AJR Am. J. Roentgenol. 143, 729-730 (1984).

31. H. S. Glazer, J. K. Lee, R. G. Levitt, J. P. Heiken, D. Ling, W. G. Totty, D. M. Balfe, B. Emani, T. H. Wasserman, W. A. Murphy, Radiation fibrosis: differentiation from recurrent tumor by MR imaging. Radiology 156, 721-726 (1985).

32. W. T. Yuh, T. J. Barloon, W. J. Sickels, E. V. Kramolowsky, R. D. Williams, Magnetic resonance imaging in the diagnosis and followup of idiopathic retroperitoneal fibrosis. J. Urol. 141, 602-605 (1989).

33. L. Arrive, H. Hricak, N. J. Tavares, T. R. Miller, Malignant versus nonmalignant retroperitoneal fibrosis: differentiation with MR imaging. Radiology 172, 139-143 (1989). 
34. L. Marti-Bonmati, A. Talens, J. del Olmo, A. de Val, M. A. Serra, J. M. Rodrigo, A. Ferrandez, V. Torres, M. Rayon, J. S. Vilar, Chronic hepatitis and cirrhosis: evaluation by means of MR imaging with histologic correlation. Radiology 188, 37-43 (1993).

35. D. G. Mitchell, Focal manifestations of diffuse liver disease at MR imaging. Radiology 185, 1-11 (1992).

36. D. D. Stark, N. M. Bass, A. A. Moss, B. R. Bacon, J. H. McKerrow, C. E. Cann, A. Brito, H. I. Goldberg, Nuclear magnetic resonance imaging of experimentally induced liver disease. Radiology 148, 743-751 (1983).

37. C. Thomsen, P. Christoffersen, O. Henriksen, E. Juhl, Prolonged T1 in patients with liver cirrhosis: an in vivo MRI study. Magn. Reson. Imaging 8, 599-604 (1990).

38. The Clinical NMR Group, Magnetic resonance imaging of parenchymal liver disease: a comparison with ultrasound, radionuclide scintigraphy and $\mathrm{x}$-ray computed tomography. Clin. Radiol. 38, 495-502 (1987).
39. G. Elizondo, R. Weissleder, D. D. Stark, J. Guerra, J. Garza, C. J. Fretz, L. E. Todd, J. T. Ferrucci, Hepatic cirrhosis and hepatitis: MR imaging enhanced with superparamagnetic iron oxide. Radiology 174, 797-801 (1990).

40. O. Clement, G. Frija, C. Chambon, E. Schouman-Clayes, J. F Mosnier, M. F. Poupon, B. Balkau, Liver tumors in cirrhosis: experimental study with SPIO-enhanced MR imaging. $R a-$ diology 180, 31-36 (1991).

41. M. Brauer, S. Locke, Proton magnetic resonance imaging and phosphorus-31 magnetic resonance spectroscopy studies of bromobenzene-induced liver damage in the rat. Magn Reson. Imaging 10, 257-267 (1992).

42. V. Rajanayagam, R. R. Lee, Z. Ackerman, W. G. Bradley, B. D. Ross, Quantitative P-31 MR spectroscopy of the liver in alcoholic cirrhosis. J. Magn. Reson. Imaging 2, 183-190 (1992).

43. J. D. Hazle, P. A. Narayana, H. A. Dunsford, Chronic carbon tetrachloride and phospholipase $\mathrm{D}$ hepatotoxicity in rat: in vivo $1 \mathrm{H}$ magnetic resonance, total lipid analysis, and histology. Magn. Reson. Med. 15, 211-228 (1990). 